\title{
Preoperative trepanation and drainage for acute subdural hematoma: Two case reports
}

\author{
TIANSHU LU ${ }^{1,2}$, JINGYU GUAN ${ }^{2}$ and CHUNLI AN ${ }^{1}$ \\ ${ }^{1}$ Department of Pathogen Biology, School of Basic Medicine, China Medical University; \\ ${ }^{2}$ General Hospital of Shenyang Military Region, Shenyang, Liaoning 110001, P.R. China
}

Received July 3, 2014; Accepted March 11, 2015

DOI: $10.3892 / \mathrm{etm} .2015 .2456$

\begin{abstract}
Craniotomy is frequently used for the treatment of acute subdural hematoma; however, it the procedure exhibits a high mortality rate. Preoperative trepanation and drainage in an emergency ward may reduce intracranial pressure, shorten operation time and lower patient mortality, and is thus applicable to the treatment of acute subdural hematoma. The present study reports the cases of two elderly patients that benefitted from trepanation and drainage of an acute subdural hematoma. In each case, the family members of the patients refused to consent to a craniotomy; thus, burr-hole drainage was selected as an alternative option for relieving intracranial pressure. The risks require careful evaluation when considering whether trepanation with drainage is an option for a patient. Following treatment, the two cases were cured and discharged on days 48 and 18 after admission, respectively. The present case studies indicate that trepanation with drainage may be a promising approach for reducing craniotomy-associated mortality and closely monitoring condition variation in elderly patients. Following trepanation with drainage, certain patients do not undergo a craniotomy.
\end{abstract}

\section{Introduction}

Acute subdural hematoma is a major life-threatening condition with a mortality rate of $60-80 \%$ (1). Subdural hematomas require immediate and aggressive treatment. The main surgical approach is to perform a craniotomy, which involves removing part of the skull, followed by drainage of the hematoma and reattachment of the skull fragment (2). Decompressive craniectomy also involves removing part of the skull to relieve intracranial pressure; however, the part of the skull that is removed is not generally replaced (2). Although craniotomies and craniectomies are frequently used for the treatment of

Correspondence to: Professor Chunli An, Department of Pathogen Biology, School of Basic Medicine, China Medical University, 92 Beier Road, Shenyang, Liaoning 110001, P.R. China

E-mail: cmucl@126.com

Key words: preoperative trepanation acute subdural hematomas, a major limitation of their use is that these surgical techniques are associated with high mortality rates $(3,4)$. Preoperative trepanation and drainage, in which a burr hole is created in the skull to reduce pressure, may improve surgical outcomes or, in some cases, reduce the need for follow-up surgical care (5). This approach may be particularly valuable for patients who present with comorbidities or cannot endure highly invasive surgical procedures. In the present study, two cases of elderly patients with acute subdural hematomas treated by preoperative trepanation and drainage are reported.

\section{Case reports}

Case 1. An 86-year-old male patient developed a sudden headache followed by nausea and vomiting with gradual loss of consciousness. In transit to the Shenyang General Hospital (Shenyang, China), the patient developed cerebral herniation with right and left mydriases of $6.0 \mathrm{~mm}$ and $2.0 \mathrm{~mm}$, respectively. The patient was initially admitted to the Emergency Department for acute subdural hematoma and later transferred to the intensive care unit of the Neurological Department. The patient was deeply comatose at the time of admission with a Glasgow Coma Scale (GCS) score of 5, with no eye movement or light reflex. The left pupil diameter was $2.0 \mathrm{~mm}$ and the right pupil diameter was $5.5 \mathrm{~mm}$. Limb-muscle strength was grade II with normal muscle tension (MRC grading system) (6), and a pathological reflex was not elicited. Computed tomography (CT) images of the head are shown in Fig. 1.

Due to the instability of the vital signs, an emergency surgery was required to remove the hematoma. Family members of the patient did not support a decompressive craniectomy with general anesthesia being performed but agreed to burr-hole drainage for subdural hematoma on the right frontal, temporal and parietal regions using local anesthesia.

After informed consent was obtained from the patients' families, local anesthesia was administered and 1.0-cm surgical incisions were created at the centers of the thickest hematoma regions in the right parietal and temporal lobes. Two 1-cm burr holes were drilled until an outflow of dark red, bloody fluid was achieved. A drainage tube (10\#; diameter, $3 \mathrm{~mm}$ ) was placed into the hematomal cavity and the incision was subsequently sutured and closed. The drainage tube was connected to a sterile drainage bag, and the incision was covered with sterile 


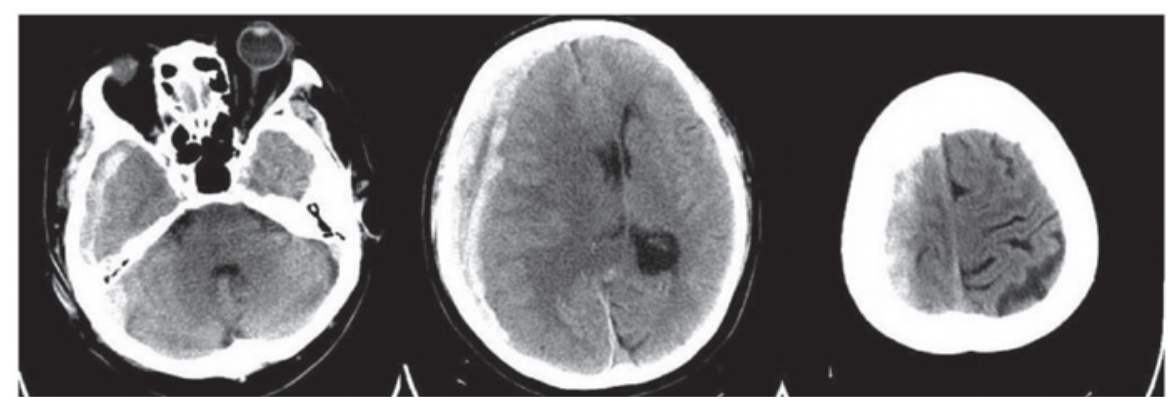

Figure 1. Preoperative computed tomography (CT) images of the head for case 1. Head CT scans reveal visible crescent-shaped, high-density regions in the temporal, occipital and parietal lobes with uneven density. Cerebral sulci and fissures over the right hemisphere are shallow or have disappeared due to pressure on the brain tissue. Pressure on the right ventricle is reduced, causing a local midline shift to the left.

dressing. The postoperative drainage tubes were patent and the patient exhibited stable vital signs following surgery. The GCS score was 4 and the pupil diameters remained unequal at 6.0 and $2.0 \mathrm{~mm}$ in the right and left eye, respectively. The light reflex remained absent. A postoperative subdural injection of urokinase $\left(5 \times 10^{4} \mathrm{IU}\right)$ accelerated the dissolution of the intracranial hematoma. The postoperative $\mathrm{CT}$ images are presented in Fig. 2.

On postoperative day 1 , the patient was comatose with a GCS score of 5. The patient showed no eye movement and had retarded light reflex in the left pupil at $2.0 \mathrm{~mm}$, and areflexia in the right pupil at $5.0 \mathrm{~mm}$. The dehydration drug Mannitol (Shanghai Baxter Healthcare Co., Ltd., Shanghai, China) was administered in three 250-ml doses and urokinase was injected into the secondary hematoma cavity to drain the hematoma. The patient was drowsy on postoperative day 3 and pupil diameters remained unequal at $2.0 \mathrm{~mm}$ in the left eye and $6.0 \mathrm{~mm}$ in the right eye. Furthermore, the light reflex remained retarded in the left pupil with areflexia in the right pupil. On postoperative day 4 , the patient exhibited a degree of unconsciousness with a GCS score between 5-8. Urokinase was injected into the hematomal cavity. CT images of the head on postoperative day 4 are shown in Fig. 3A. Hypoalbuminemia and a significantly increased inflammatory cell count were noted on postoperative day 5. Elevated sodium and reduced potassium levels were also observed but were corrected. Sodium levels were corrected by administering the patient drinking water, while potassium levels were normalized via the intravenous administering of potassium. CT images showed no abnormalities on postoperative day 6 (Fig. 3B). On postoperative day 9, the patient exhibited a degree of unconsciousness (GSC score 5-8) and had excessive levels of sputum. A percutaneous tracheal atherectomy was performed and head CT scans were obtained (Fig. 3C). On day 26 following admission, the patient showed faint signs of consciousness with stable vital signs and head images. However, serious lung inflammation (Fig. 4) required continuous intervention with three 4.5-g doses of piperacillin/tazobactam (Qingzhou Yaowang Medicine Co., Ltd., Weifang, Shandong, China) by the Department of Respiratory Medicine. The patient regained consciousness, showed signs of recovery and was discharged on day 48 after admission.

Case 2. A 74-year-old male patient developed transient chest pain and was admitted to the Shenyang General Hospital for further treatment following an unspecified drug treatment at a different hospital. The patient had been diagnosed with coronary artery disease and myocardial infarction with an acute non-ST segment elevation 3 years previously. There was no history of hypertension, diabetes mellitus or cerebrovascular disease. The patient was diagnosed with coronary atherosclerotic heart disease with unstable angina, old anterior myocardial infarction and cardiac function level II (New York Heart Association class) (7) at the time of admission. A single 100-mg dose of aspirin (Bayer AG, Leverkusen, Germany) was administered as an anti-platelet therapy, resulting in protection of the gastric mucosa, adjustment of the levels of blood fat, improved blood circulation, controlled heart rate, decreased myocardial oxygen consumption, dilated coronary arteries and reduced cardiac stress.

Coronary angiography showed $99 \%$ occlusion of the proximal and middle right coronary artery with the appearance of bridge collaterals on day 3 following admission. Furthermore, complete $(100 \%)$ occlusion of the distal left circumflex artery and $90-95 \%$ stenosis of the proximal and middle left anterior descending artery was observed. The Thrombolysis in Myocardial Infarction (TIMI) score was 2-3 (8). Septal branches extended to the left circumflex artery and obtuse marginal branches to form a coarse two-level collateral circulation. The family of the patient requested coronary-artery bypass grafting.

The patient was scheduled for surgery and preoperative preparations by the Department of Cardiac Surgery on day 5 after admission. However, the patient developed a fever of $37.6^{\circ} \mathrm{C}$ and $0.5 \mathrm{~g}$ paracetamol (Southwest Pharmaceutical Co., Ltd., Chongqing, China) was administered orally as an antipyretic. The patient developed sudden slurring of speech, lethargy, and right-limb movement disorder by the next day (day 6 after admission). Cranial CT scans showed the presence of a left-sided subdural hematoma (Fig. 5). The patient was transferred to the intensive care unit of the Neurosurgery Department. The family of the patient did not allow the medical team to perform a decompressive craniectomy with general anesthesia. However, following additional discussions, the family consented to burr-hole drainage with local anesthesia as a treatment for the left subdural hematoma.

The patient showed faint consciousness on day 6 after admission. Burr-hole drainage was performed and surgical incisions were created at the centers of the thickest regions of the hematoma in the left parietal lobe to expose the dura, 

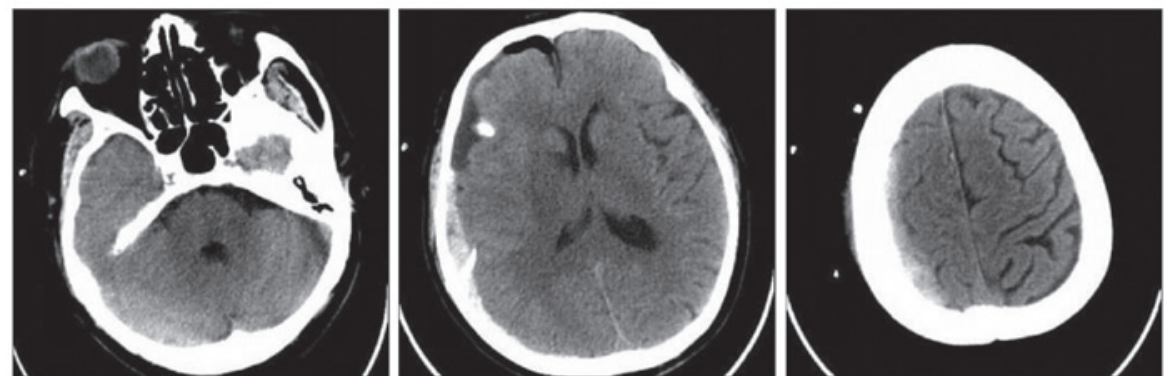

Figure 2. Computed tomography (CT) images for case 1 on the day of surgery. Head CT scans show crescent-shaped, mixed shadows of high and low densities in the right frontal, temporal and parietal lobes. The drainage tube extends outside the brain. The right ventricle is smaller and the bilateral corona radiata show small, low-density shadows with clear boundaries. Certain sulci and fissures remain enlarged, but no midline shift has occurred.
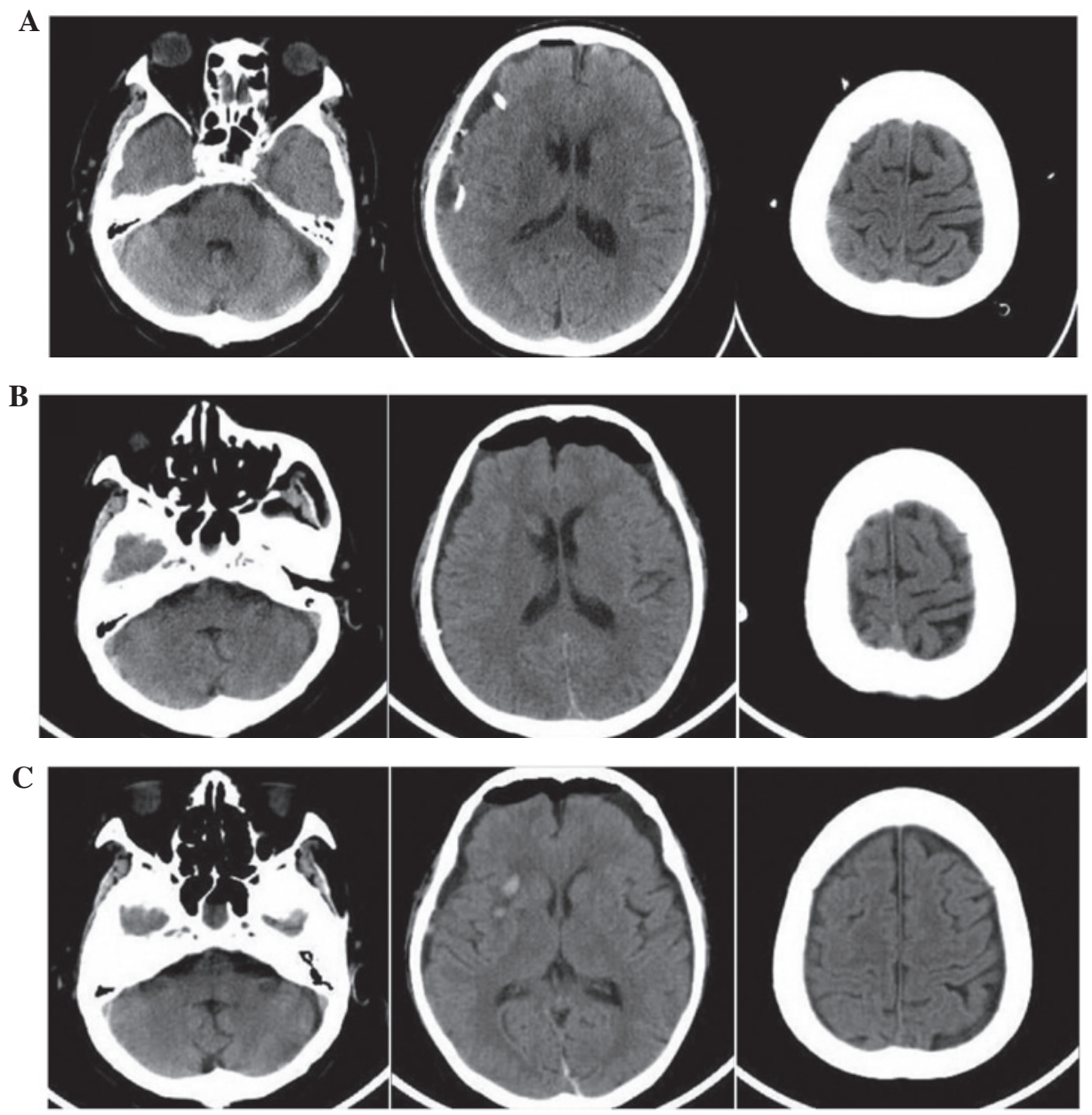

Figure 3. Postoperative computed tomography (CT) images for case 1. Head CT scans on postoperative (A) day 4 show the subdural hematoma has disappeared and the right ventricle is restored with a normal midline, (B) day 6 exhibit an evident reduction of blood loss and (C) day 9 present with no evidence hematocele.
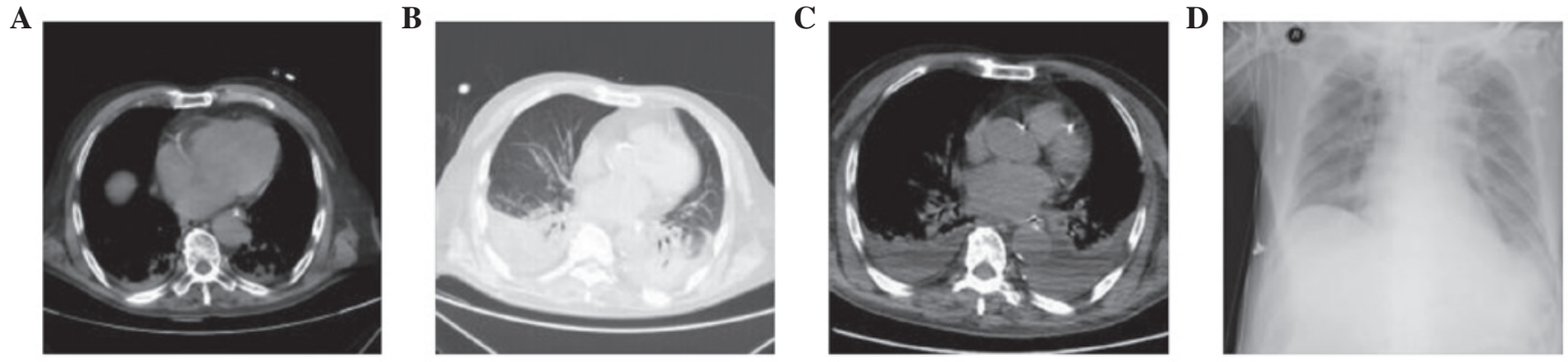

Figure 4. Lung computed tomography images for case 1 on days (A) 9, (B) 20 and (C) 25 after admission. (D) Chest X-ray on day 32 after admission. 


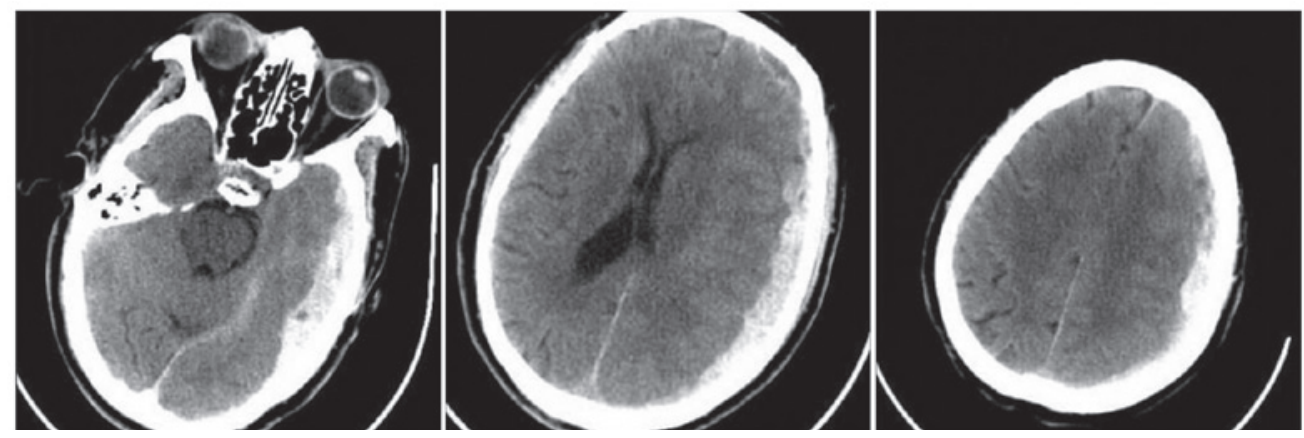

Figure 5. Preoperative computed tomography images of the head for case 2, displaying left-sided subdural hemorrhage.
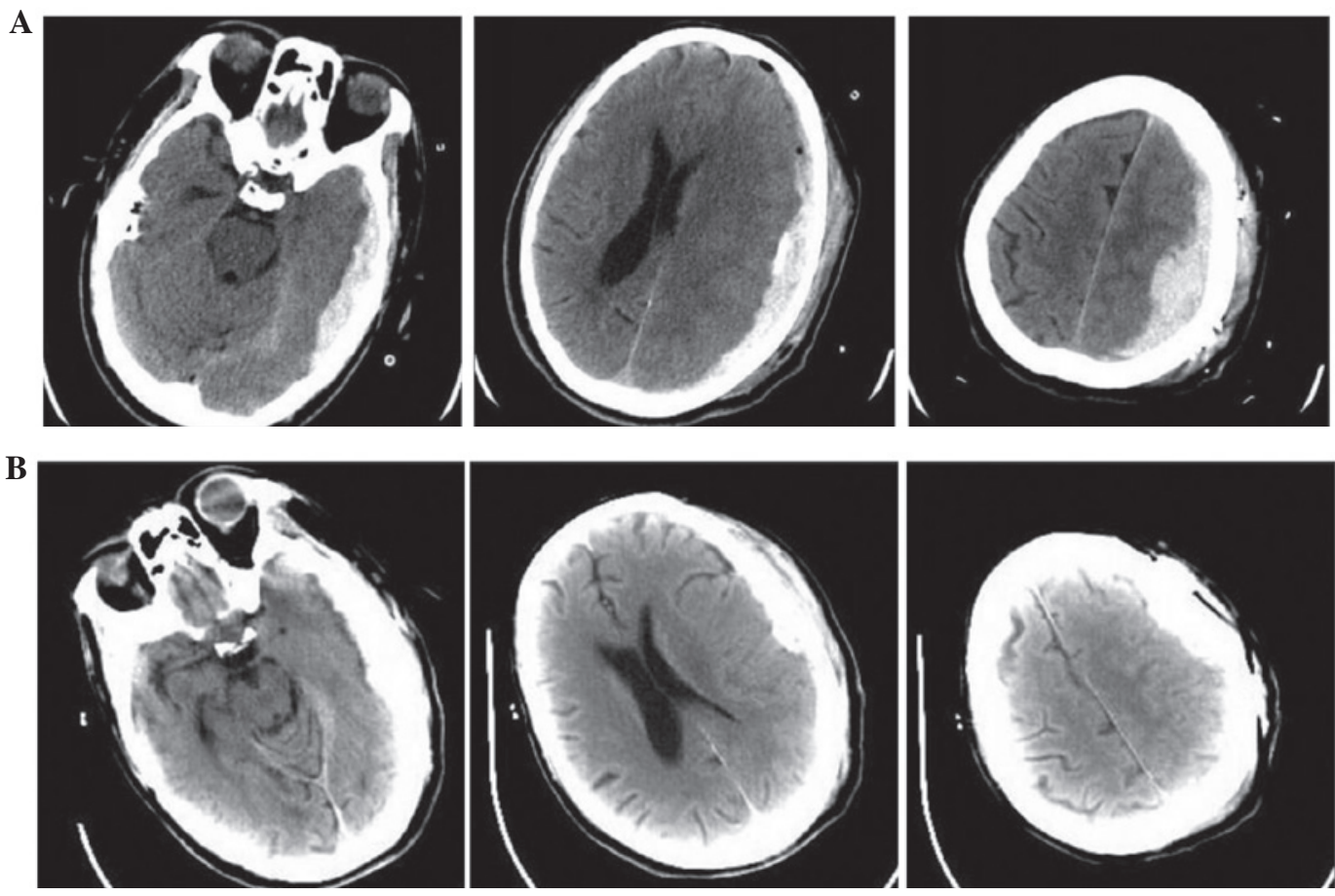

Figure 6. Postoperative computed tomography (CT) images of the head for case 2. (A) Head CT images immediately following surgery, with drainage tube located in the hematoma cavity. (B) Head CT images on postoperative day 5, with notably reduced hematoma volume.
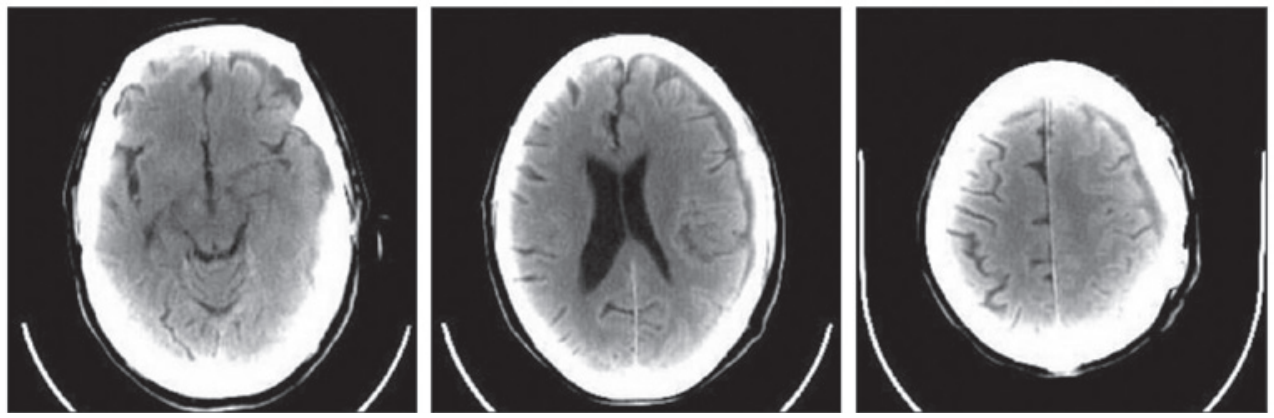

Figure 7. Computed tomography images of the head for case 2 on postoperative day 18 at the time of discharge, with the majority of the hematoma cleared.

which was dark blue and showed a high degree of tension. Following fulguration, a cross-shaped incision was created to open the dura until an outflow of $20 \mathrm{ml}$ of dark red, bloody fluid was achieved. A 10\# pipe was placed into the hematomal cavity followed by washout with normal saline. The incision was sutured and closed layer by layer. The drainage tube was rinsed with an ample amount of normal saline until the saline was clear; the tube was subsequently connected to a sterile drainage bag. A further incision was created in the left temporal lobe as described above. The patient was in a coma on the day of surgery with a GCS score of 7 and equal pupil diameters of $1.5 \mathrm{~mm}$ each. Furthermore, the patient exhibited 
a retarded light reflex, tingling of the limbs and bilateral negative pathological signs. Postoperative seizures occurred several times and valproate and sedative drugs (propofol, 20 mg/h; Fresenius Kabi, Bad Bomburg, Germany) were administered in combination due to concerns of vasospasms and transient convulsions secondary to reduced intracranial pressure. Postoperative CT imaging was performed (Fig. 6A).

The GCS score was 7 on days 1-4 following surgery, 9 on days 5-12 and ranged from 10 to 14 on days 12-18. Subdural injection of urokinase was performed on postoperative days 1 and 2 to accelerate clot dissolution. The patient received a tracheotomy on postoperative day 3. On postoperative day 5 , the white-blood cell (WBC) count was $28.6 \times 10^{9} / 1$. The subdural hematoma was clearly reduced on CT scans (Fig. 6B); thus, the drainage tube was removed. Serum sodium was elevated to $150.9 \mathrm{mmol} / \mathrm{l}$ on postoperative day 6 ; serum potassium was $2.86 \mathrm{mmol} / \mathrm{l}$; and the WBC count was $29.7 \times 10^{9} / 1$. The patient was required to drink more water to reduce the levels of serum in the blood, and potassium levels were increased by intravenuous injections. Sputum cultures showed the presence of Acinetobacter baumannii and Klebsiella pneumoniae. A total of $2.0 \mathrm{~g}$ of the antibiotic meropenem was administered every $8 \mathrm{~h}$ for 2 weeks. Routine blood tests performed on postoperative day 7 showed reduced serum levels of prealbumin (129 mg/l) and albumin (35.3 g/l), increased serum levels of sodium $(157.1 \mathrm{mmol} / \mathrm{l})$, and an increased neutrophil percentage $(87.9 \%)$ and neutrophil count (21.0x10 $/ 1)$. Routine blood tests on postoperative day 8 showed a decreased WBC count $\left(13.6 \times 10^{9} / 1\right)$ as well as increased levels of globulin $\mathrm{f}(30.80 \mathrm{~g} / \mathrm{l})$, sodium $(150.4 \mathrm{mmol} / \mathrm{l})$ and potassium ( $3.25 \mathrm{mmol} / \mathrm{l})$. Potassium supplements were administered to the patient and the hypernatremia was corrected. On postoperative day 9, the WBC count remained at 13.6x10 $/ 1$; however, the levels of sodium and potassium ions returned to normal. The postsurgical wound healed well. The WBC count gradually returned to normal between postoperative days 10-18. The patient was discharged on postoperative day 18; head CT images performed at the time of discharge are shown in Fig. 7.

\section{Discussion}

The present study reported two cases in which elderly patients benefited from trepanation and drainage of an acute subdural hematoma. In all cases, the families of the patients refused to consent to surgery; thus, burr-hole drainage was selected as an alternative option for relieving intracranial pressure. A number of studies have demonstrated that the timing of surgical intervention is a major determinant of the outcome of subdural hematoma treatment $(4,9)$. The recommended timing for evacuation of a hematoma is within $4 \mathrm{~h}$ of onset; delays past this critical window of time are associated with neuronal loss and poorer outcomes in a number of neurological conditions, including subdural hematomas and strokes $(4,10)$. Trepanation and drainage is a rapid method for stabilizing a patient with an early-stage subdural hematoma who is unable to receive surgery in a timely manner. However, drainage through a burr hole may only be an option during the first few hours of developing a subdural hematoma, as coagulation may eventually prevent blood flow through the burr hole (11). Patients who are cotreated with anticoagulants may show more favorable treatment results with trepanation, as these medications may maintain steady blood flow and an open drainage tube, thereby relieving intracranial pressure (12). In a previous study, the insertion of a subdural evacuating port system with subsequent drainage of a subdural hemorrhage and a craniotomy resulted in a favorable neurological outcome and recovery for the patient (11). Thus, the option of trepanation and drainage may be suitable for patients who cannot immediately receive surgery. However, the benefit must always be weighed against potential disadvantages, which include the potential for infection associated with the burr hole, as shown in the present study with case 2 . In case 2 , the adverse event was successfully managed with medication. Another potential disadvantage of burr-hole drainage is that it may further delay surgery (12). These risks need to be weighed carefully when considering whether trepanation with drainage is an option for a patient.

In the current study, case 1 presented a relatively low GCS score of 4 at the time of admission to the hospital. Initial GCS scores at the time of admission have previously been associated with mortality rate (13). In one study, GCS scores $<3$ were associated with a $93 \%$ mortality rate in patients with acute subdural hematomas (14). Patients in the study who had GCS scores between 4 and 6 demonstrated mortality rates ranging from 45 to $67 \%$, and all patients with GCS scores $\geq 7$ survived (14). Another study reported a $74 \%$ mortality rate for those with GCS scores between 3 and 5 (15). In addition to GCS score, age is a major determinant of outcome from acute subdural hematoma. It is estimated that the probability of a poor outcome increases by $40-50 \%$ with every decade of life (13). Furthermore, mortality appears to be 4 times more likely in patients who are $\geq 65$ years of age than in adults who are $<40$ years of age (13). Another factor that may favorably influence outcome is whether the patient is treated in a dedicated neurosurgical department (13), as occurred in the cases discussed in the present study. Based on the associations between age and GCS score with mortality rate, the favorable outcomes of the cases reported in the current study suggest that trepanation with drainage may be a promising treatment approach for elderly patients, including those with low GCS scores. Future trials should be performed to determine if preoperative burr-hole drainage can effectively improve the outcome and reduce the mortality rate in elderly patients with acute subdural hematomas.

\section{References}

1. Park JY, Moon KS, Lee JK and Jeung KW: Rapid resolution of acute subdural hematoma in child with severe head injury: a case report. J Med Case Rep 7: 67, 2013.

2. Chen SH, Chen Y, Fang WK, Huang DW, Huang KC and Tseng SH: Comparison of craniotomy and decompressive craniectomy in severely head-injured patients with acute subdural hematoma. J Trauma 71: 1632-1636, 2011.

3. Li LM, Kolias AG, Guilfoyle MR, Timofeev I, Corteen EA, Pickard JD, Menon DK, Kirkpatrick PJ and Hutchinson PJ: Outcome following evacuation of acute subdural haematomas: a comparison of craniotomy with decompressive craniectomy. Acta Neurochir (Wien) 154: 1555-1561, 2012.

4. Paci GM, Sise MJ, Sise CB, Sack DI, Shackford SR, Kureshi SA, Osler TM, Yale RS, Riccoboni ST, Peck KA and O'Reilly EB: Preemptive craniectomy with craniotomy: what role in the management of severe traumatic brain injury? J Trauma 67: 531-536, 2009.

5. Mondorf Y, Abu-Owaimer M, Gaab MR and Oertel JM: Chronic subdural hematoma - craniotomy versus burr hole trepanation. $\mathrm{Br}$ J Neurosurg 23: 612-616, 2009 
6. Chisari C, Bertolucci F, Dalise S and Rossi B: Chronic muscle stimulation improves muscle function and reverts the abnormal surface EMG pattern in myotonic dystrophy: A pilot study. J Neuroeng Rehabil 10: 94, 2013.

7. Johnson MJ, Bland JM, Davidson PM, et al: The relationship between two performance scales: New York Heart Association Classification and Karnofsky Performance Status Scale. J Pain Symptom Manage 47: 652-658, 2014

8. Amin ST, Morrow DA, Braunwald E, et al: Dynamic TIMI risk score for STEMI. J Am Heart Assoc 2: e003269, 2013.

9. Bulters D and Belli A: A prospective study of the time to evacuate acute subdural and extradural haematomas. Anaesthesia 64: 277-281, 2009.

10. Saver JL: Time is brain - quantified. Stroke 37: 263-266, 2006.

11. Flint AC, Gean AD, Manley GT, et al: Temporizing treatment of hyperacute subdural hemorrhage by subdural evacuation port system placement. J Neurosurg 115: 844-848, 2011.
12. Sagher O: Acute subdural hematoma. J Neurosurg 115: 842 , 2011.

13. Leitgeb J, Mauritz W, Brazinova A, Janciak I, Majdan M, Wilbacher I and Rusnak M. Outcome after severe brain trauma due to acute subdural hematoma. J Neurosurg 117: 324-333, 2012.

14. Hatashita S, Koga N, Hosaka Y and Takagi S. Acute subdural hematoma: severity of injury, surgical intervention and mortality. Neurol Med Chir (Tokyo) 33: 13-18, 1993.

15. Gennarelli TA, Spielman GM, Langfitt TW, Gildenberg PL, Harrington T, Jane JA, Marshall LF, Miller JD and Pitts LH. Influence of the type of intracranial lesion on outcome from severe head injury. J Neurosurg 56: 26-32, 1982. 